\title{
Active Control of the Sound Power Scattered by a Locally-Reacting Sphere
}

\author{
Stephen J. Elliott, ${ }^{1,}$ a) Mihai Orita, ${ }^{1}$ and Jordan Cheer $^{1}$ \\ Institute of Sound and Vibration Research, University of Southampton, Southampton, SO17 1BJ
}

Active control of the sound power scattered by a sphere is theoretically investigated using spherical harmonic expansions of the primary and secondary fields. The sphere has a surface impedance that is uniform, real and locally-reacting, while being subjected to an incident monochromatic plane wave. The scattered power is controlled with a number of monopole sources, initially on the surface of the sphere, and is expressed as the sum of squared amplitudes of the spherical harmonics, due to both the scattered and control fields. This quadratic function is minimized to identify the optimal strengths for different numbers of control sources. At low frequencies, the scattered field is dominated by the first few spherical harmonic terms, and its power can be significantly reduced with a single controlling monopole, for a soft or absorbent sphere, and with two monopoles for a hard sphere. The number of secondary sources required to significantly attenuate the scattered field at higher frequencies is found to be proportional to the square of the frequency and the attenuation also falls off rapidly if the secondary sources are moved away from the surface of the sphere, no matter what its surface impedance.

(C)2020 Acoustical Society of America. [http://dx.doi.org(DOI number)]

$[\mathrm{XYZ}]$

Pages: 1-13

\section{INTRODUCTION}

When an incident travelling disturbance impinges on an obstacle, it can create reflection, absorption, refraction and diffraction. The resulting artefacts generated in the medium exterior to the obstacle are referred to as scattering. The understanding and control of acoustic scattering is important in a number of practical applications. One example is binaural sound reproduction, where the physical presence of the head plays a major role on the perceived sound ${ }^{1,2}$. Another example is acoustic cloaking of objects ${ }^{3-6}$, which is important in scenarios involving detection.

Sound scattering can be controlled through both passive and active methods. As with other acoustic and vibration control problems, using traditional passive absorbing elements imposes limitations on performance, particularly at low frequencies. As a result, alternatives such as active control have been investigated. The earliest published work on controlling scattered sound is that of Malyuzhinets, Jessel and Mangiante from the 1960s and $1970 \mathrm{~s}$, as summarised $\mathrm{in}^{3}$. This theoretical result relies on surrounding the scatterer with four Huygens surfaces that are themselves acoustically transparent. A continuous array of pressure and velocity sensors placed on two inner surfaces extract the scattered field from the total field based on the Helmholtz-Huygens integral. A continuous array of monopole and dipole sources placed on two outer surfaces then radiate a counter-phase version of the scattered field in the exterior region, such

a) S.j.elliott@soton.ac.uk that perfect suppression is achieved ${ }^{3}$. However, the tactic is limited in practice by the need for acoustic transparency of the four surfaces and by the need to use a finite number of transducers ${ }^{4}$. The ideal suppression approach described above was used in the work of Vasquez et $a l^{5}$ and Norris et $a l^{6}$ to develop theoretical results and numerical simulations for active acoustic cloaks of $2 \mathrm{D}$ obstacles. Their strategy relies on obtaining similar cancellation of the scattered field to that of the continuous layers of monopoles and dipoles, with a small number of surrounding multipole sources.

A different active control method from that of Malyuzhinets, Jessel and Mangiante is to attenuate the scattered field by using a finite number of sources to minimize the scattered power. This is a better representation of what can be achieved in practice and has been traditionally realised by minimizing the sum of the mean square acoustic pressures, measured at sensor locations around the obstacle. Such an approach is covered in the textbooks ${ }^{3}$ and ${ }^{7}$, and it is the basis of a number of subsequent publications: ${ }^{4,8-11}$. Of particular note to the present paper is the approach of Liu et $\mathrm{al}^{10}$, where a spherical harmonic representation of the scattered field for an acoustically hard obstacle was used in conjunction with the control strategy of minimizing the sum of squared pressures.

Spherical harmonics are orthogonal functions defined on a spherical surface and appear as components of the solutions to the Helmholtz equation when using spherical polar coordinates. The use of spherical harmonic series expansions has gained particular attention in recent decades as a result of developments in 3D surround sound and audio reproduction applications, such as ${ }^{12}$. A spher- 
ical shape is convenient because of the inherent theoretical and practical advantages resulting from its symmetry and relatively smooth curvature. Furthermore, orthogonality is a desirable property since it allows the analysis and manipulation of a field to be carried out using individual, uncoupled components. This is useful for the purpose of active control because it creates the possibility of cancelling one or more unwanted components without unduly affecting the others, as long as the secondary field can be designed to match that unwanted set of components.

This paper considers the active control of sound scattered from objects whose acoustic behaviour is defined by a locally reacting impedance on their surface, subject to an incident tonal plane wave. In particular we will consider a spherical harmonic formulation for the active control of the sound power scattered by a sphere with a real, uniform surface impedance. The minimisation problem is formulated in terms of the sum of the squared amplitudes of a finite orthogonal series for the scattered power, which are the spherical harmonic components in this case. This formulation allows physical insight into the mechanisms of active control of the acoustic scattering from a 3D body, in terms of performance limits and secondary source positioning. Apart from the formulation of active control in terms of an orthogonal series, the main contributions of this paper are the insight into the form of scattering behaviour with different surface impedances and the analytic results for the attenuation achieved at low frequencies with one or two secondary sources. In addition a simple result is derived for the number of sources required for effective active control at higher frequencies, whether the sources are on or away from the surface of the sphere. A preliminary version of this work was presented in ${ }^{13}$.

The formulation of the scattering problem for a locally reacting sphere is first reviewed in Section II. Section III discusses the spherical harmonic decomposition of the sound field generated by secondary monopole sources on the surface of such a sphere, and also the limiting behaviour in the low-frequency, Rayleigh limit, where active control is most effective. Section IV then describes the results of active control simulations using various arrangements of surface monopoles as secondary sources and discusses the required number of secondary sources to achieve a given level of active control. Finally, Section $V$ considers the performance when the secondary sources are moved away from the surface of the scatterer and Section VI provides some discussion and conclusions, particularly the generalisation of the spherical harmonic formulation using radiation modes for other shapes of scatterer.

\section{SPHERICAL HARMONIC FORMULATION OF SCAT- TERING FROM A LOCALLY REACTING SPHERE}

Rayleigh ${ }^{14,15}$ first described how the scattered sound field from a rigid sphere could be obtained by satisfying the boundary conditions on the surface of the sphere us- ing a spherical harmonic decomposition of the incident and scattered fields. The formulation has been extended, for example by Godin ${ }^{16}$ and $\mathrm{Mao}^{17}$, for the case of scattering from a locally reacting sphere when subject to an incident field due to a monopole source a finite distance away. If the monopole is far from the sphere, this approach can be used to calculate the scattered field due to an incident plane wave, and if the monopole is on the surface of the sphere, it can be used to calculate the sound field generated by a surface secondary source. This general theoretical formulation is briefly reviewed in appendix A, for completeness and also since we assume an $e^{j \omega t}$ convention for the time variation of the harmonic sound field in this paper, where $\omega$ is the angular frequency, to be consistent with the active control and signal processing literature, rather than the $e^{-\mathrm{i} \omega t}$ convention adopted by Godin ${ }^{16}$, Williams ${ }^{18}$, and much of the cloaking literature, so that the details of the formulation are slightly different. The frequency dependence of the complex variables is suppressed for notational convenience.

The complex scattered acoustic field, $p_{s}$, at position $(r, \theta, \varphi)$ due to a harmonic plane wave of complex amplitude $p_{i}$, arriving from direction $\left(\theta_{i}, \varphi_{i}\right)$, incident on a sphere of radius $a$ can be written as

$$
p_{s}(r, \theta, \varphi)=\sum_{n=0}^{\infty} \sum_{m=-n}^{n} a_{n m}^{s} h_{n}(k r) Y_{n}^{m}(\theta, \varphi)
$$

where $k$ is the wavenumber, $\omega / c, h_{n}(k r)$, is the complex spherical Hankel function of the second kind and $Y_{n}^{m}(\theta, \varphi)$ is a complex spherical harmonic, whose shapes in $3 \mathrm{D}$ space are illustrated in ${ }^{18}$ and $^{19}$ for example, and the complex amplitude of each term is equal to

$$
a_{n m}^{s}=-4 \pi p_{i j}{ }^{n} \bar{Y}_{n}^{m}\left(\theta_{i}, \varphi_{i}\right) A_{n}^{s}(k a),
$$

in which the overbar denotes complex conjugation. $A_{n}^{s}(k a)$ is a non-dimensional function of $k a$ given by

$$
A_{n}^{s}(k a)=\frac{j_{n}(k a)+\mathrm{j} \zeta j_{n}^{\prime}(k a)}{h_{n}(k a)+\mathrm{j} \zeta h_{n}^{\prime}(k a)},
$$

where $\zeta$ is the normalized surface impedance, which is equal to $z /(\rho c)$ where $z$ is the locally reacting impedance of the sphere and $\rho$ and $c$ are the density and speed of sound in the surrounding fluid, $j_{n}$ is a spherical Bessel function and the dashes represent the spatial derivatives of the functions with respect to $k a$. The acoustic power scattered by the sphere is then ${ }^{18}$

$$
W_{s}=\frac{1}{2 \rho c k^{2}} \sum_{n=0}^{\infty} \sum_{m=-n}^{n}\left|a_{n m}^{s}\right|^{2} .
$$

So,

$$
W_{s}=\frac{2 \pi\left|p_{i}\right|^{2}}{\rho c k^{2}} \sum_{n=0}^{\infty}(2 n+1)\left|A_{n}^{s}(k a)\right|^{2},
$$

where the closure relationship for spherical harmonics, $\sum_{m=-n}^{n}\left|\bar{Y}_{n}^{m}\left(\theta_{i}, \varphi_{i}\right)\right|^{2}=\frac{(2 n+1)}{4 \pi}$ has been used to obtain 
equation (5). If the acoustic power associated with the incident plane wave is defined as the product of the incident intensity and the cross-sectional area of the sphere,

$$
W_{i}=\frac{\pi a^{2}}{2 \rho c}\left|p_{i}\right|^{2}
$$

then the normalized scattered power can be written as

$$
\Pi_{s}=\frac{W_{s}}{W_{i}}=\frac{1}{\pi(k a)^{2}\left|p_{i}\right|^{2}} \sum_{n=0}^{\infty} \sum_{m=-n}^{n}\left|a_{n m}^{s}\right|^{2},
$$

so,

$$
\Pi_{s}=\frac{4}{(k a)^{2}} \sum_{n=0}^{\infty}(2 n+1)\left|A_{n}^{s}(k a)\right|^{2} .
$$

The normalized scattered power is equal to the scattering cross section, as used for example in ${ }^{20,21}$, divided by $\pi a^{2}$. It should be noted that although the scattered power, as defined equation (8), is a useful measure of the spaceaveraged mean square pressure that is scattered into the far field, it does not represent the actual power radiated by the sphere, which depends on the interaction between the incident and scattered fields.

Figure 1 shows the variation in the normalized scattered power, $\Pi_{s}$, with normalized frequency, $k a$, calculated using this formulation for three different values of locally reacting surface impedance. These were computed using a finite summation in equation (8), up to $n=100$, which was found to accurately represent the scattered power up to $k a=100$, since doubling the number of terms resulted in differences of less than $0.06 \mathrm{~dB}$ in the scattered power at any frequency. When the surface impedance is the same as the characteristic impedance of the fluid surrounding the sphere, so that $\zeta=1$ which is described as the " $\rho c$ " case, the normalized scattered power initially increases in proportion to $(k a)^{2}$ and has a limiting value of unity at high frequency. This is because a sound power equal to the incident sound power is absorbed by the sphere, but the sphere casts a shadow at higher frequencies. If the normalized surface impedance of the sphere is $10^{-2}$, the variation in the scattered sound power with $k a$ cannot be distinguished from that of a sphere whose surface impedance is zero over the frequency range and on the resolution of Figure 1. This finite surface impedance is used here as an example of a soft sphere, however, since a monopole placed on its surface will generate a finite external pressure field, unlike when the surface impedance is zero, as described below. In the case of the soft sphere, the scattered power is four times the incident sound power, as defined by equation (6) for small $k a^{20}$. The soft sphere scatters sound strongly at low frequencies since, to realise the pressurerelease boundary condition at the surface, the incident pressure, which is uniform over the surface of the sphere at low frequencies, can be thought of as being balanced by that due to a monopole source at the centre of the sphere, of strength $4 \pi a p_{i} /(\mathrm{j} k \rho c)$. The power radiated by such a monopole is $\pi a^{2}\left|p_{i}\right|^{2} /(\rho c)$, so that when divided by the incident sound power in equation (6), the normalized scattered power is equal to 4 . At high frequencies, the scattered power is twice the incident power, since not only is a shadow generated by the sphere, but the incident sound field is reflected off the almost-pressurerelease boundary condition at the front of the sphere.

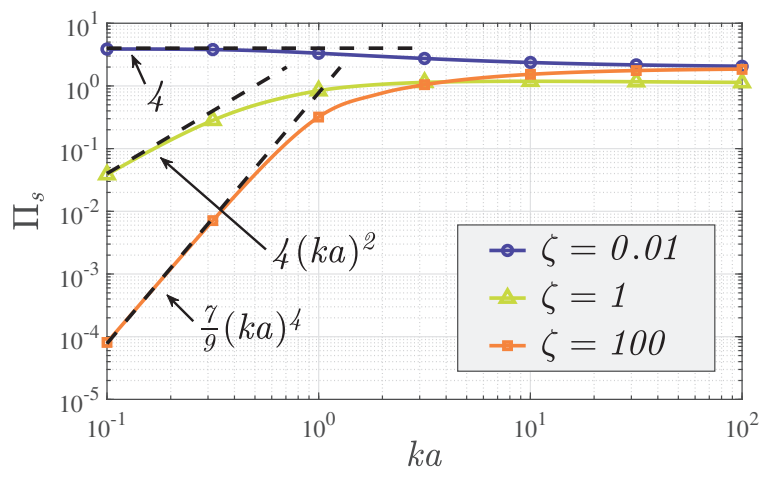

FIG. 1. The normalized scattered sound power from spheres with normalized surface impedances that are soft, $\zeta=0.01$, $\rho c, \zeta=1$, and hard, $\zeta=100$, as a function of normalized frequency $k a$. (Color online)

When the normalized surface impedance is 100 times the characteristic impedance of the surrounding fluid, the results are indistinguishable from the case when the normalized surface impedance is infinite, within the resolution used in Figure 2, and this is used here as a practical example of a hard sphere. In this case, the scattering is very small at low frequencies, with the normalized scattered sound power being proportional to $(k a)^{4}$, but at high frequencies the scattered sound power is again twice the incident sound power, since the sphere both generates a shadow and reflects the incident wave off the almostzero-velocity surface.

Figure 2 shows the spatial distribution of the instantaneous total pressure field around these three spheres, when subject to a unit amplitude plane wave from the left, for three different values of $k a$. In this case, terms up to $n=438$ were required in the summations for the incident and scattered pressures to ensure that the meansquare error was always below $0.8 \%^{22}$. The hard sphere barely affects the field at low frequencies, but at higher frequencies there is clearly a reflection from the front of the sphere and a shadow is produced behind the sphere. This reflection and shadow at higher frequencies are also seen in the case of the soft sphere, although in this case the incident field is also significantly perturbed at low frequencies. In the case of the $\rho c$ sphere, the incident field is slightly perturbed at low frequencies, and at higher frequencies it produces a shadow behind the sphere, but does not generate a reflection of the incident wave from the front of the sphere.

Further insight into the scattered field can be gained by studying the individual behaviour of the spherical harmonic components in its expansion. Figure 3 shows the variation with $k a$ of the modulus of the non-dimensional 

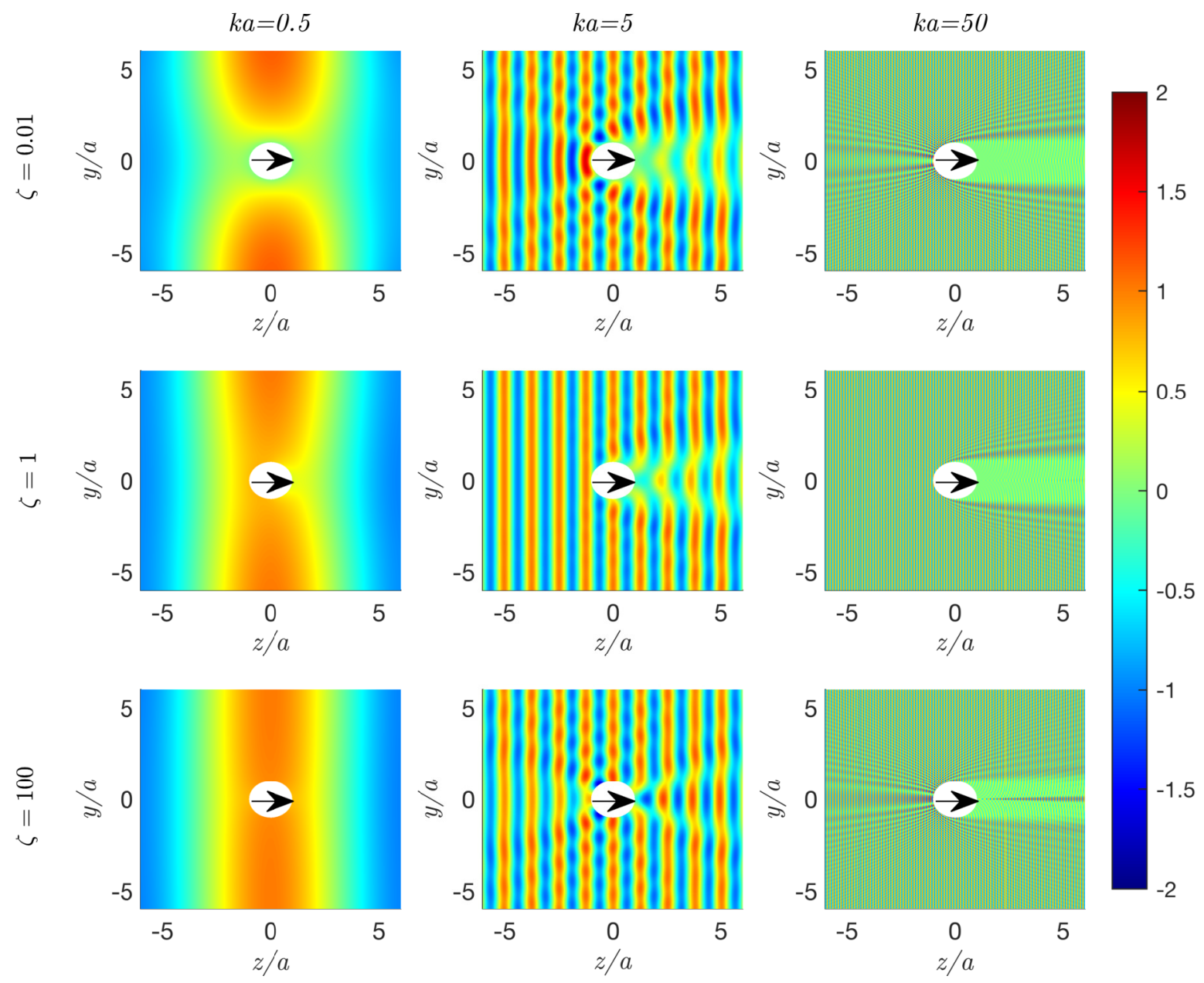

FIG. 2. The instantaneous distribution of the total pressure field, incident plus scattered, due to the interaction between a sphere having a soft, $\zeta=0.01, \rho c, \zeta=1$, or hard, $\zeta=100$, surface impedance subject to a plane wave of unit magnitude incident from below and frequency corresponding to a value of $k a$ of $0.5,5$ or 50 . (Color online) (Animations available online)

function for the scattered wave, $A_{n}^{s}(k a)$ in equation (3). The contributions of the various spherical harmonics to the overall scattered power in Figure 1 can be deduced from these parameters, whose squared quantities can be substituted into equation (8) to give the total normalized scattered power. The low frequency limits of $A_{n}^{s}$ for the spherical harmonic components of the scattered fields due to an incident plane wave, can be obtained from the small-argument expansions of the spherical Bessel and Hankel functions ${ }^{16}$, and are given, for $n=0$, by

$$
\lim _{k a \ll 1} A_{0}^{s}=\frac{(k a)^{2}(1-\mathrm{j} \zeta k a / 3)}{\zeta+\mathrm{j} k a}\left[1+O\left(k^{2} a^{2}\right)\right],
$$

and for $n \geq 1$ by

$$
\begin{aligned}
& \lim _{k a \ll 1} A_{n}^{s}= \\
& {\left[\frac{2^{n} n !}{(2 n) !}\right]^{2} \frac{(k a)^{2 n+1}}{2 n+1} \frac{k a+\mathrm{j} \zeta n}{\zeta(n+1)+\mathrm{j} k a}\left[1+O\left(k^{2} a^{2}\right)\right] .}
\end{aligned}
$$

It is clear from equation (9) that at low frequencies the $n=0$ component of the pressure scattered by a locallyreacting sphere, which is proportional to $A_{0}^{s}$, can be set to zero if $\zeta=3 / \mathrm{j} k a$. The overall acoustic impedance of the sphere is thus equal to $1 / \mathrm{j} \omega C$ where $C=\frac{4}{3} \pi a^{3} / \rho c^{2}$ i.e. the compliance of a sphere of air equal to the size of the scattering object.

The low frequency limits of these coefficients for $n=0, n=1$ and $n=2$ are given in Table I for the soft, i.e. $\zeta \ll k a, \rho c$, i.e. $\zeta=1$, and hard, i.e. $\zeta \gg k a$, spheres, where it is also assumed that $k a \gg 3 / \zeta$ for the hard sphere in the case of equation (9). By examining the numerator of equation (10), it can be seen that the lower limiting frequency below which the $n$-th spherical harmonic of the sphere with a normalized surface impedance of $1 / 100$ no longer behaves like a perfectly soft sphere corresponds to a value of $k a$ equal to about $(n+1) / 100$, which is well outside the range plotted in Figure 3. Sim- 


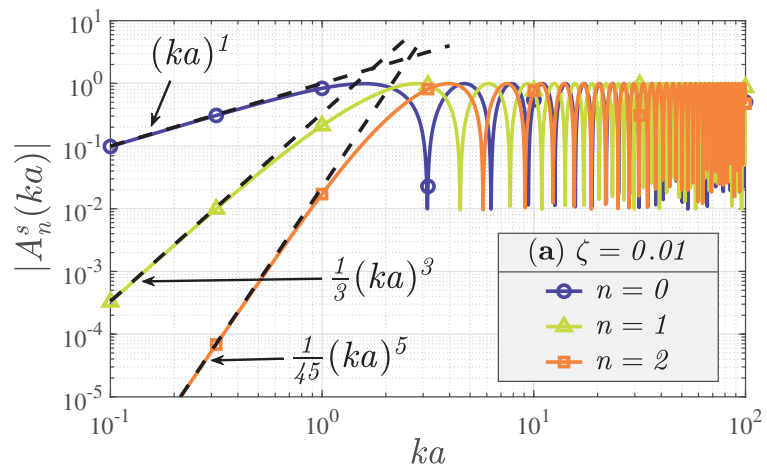

(a)

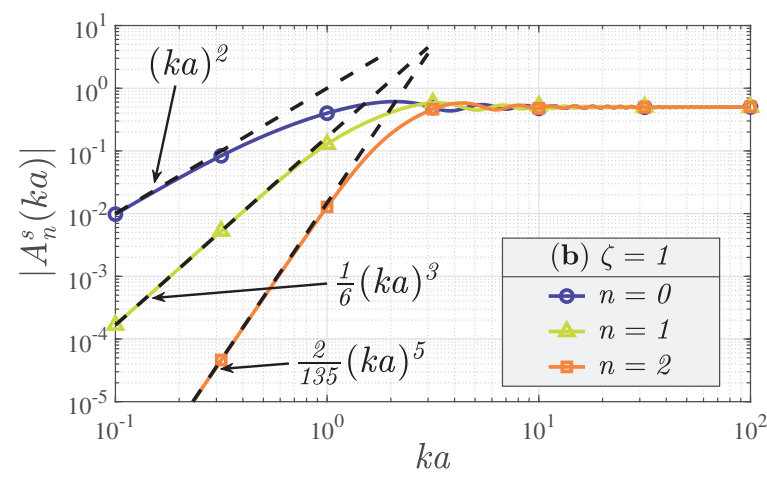

(b)

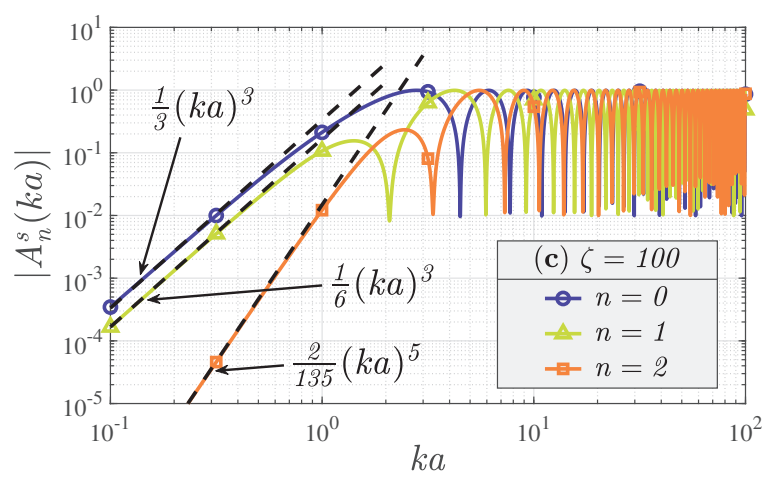

(c)

FIG. 3. The variation of the magnitude of the nondimensional spherical harmonic amplitudes, $A_{n}^{s}(k a)$ in equation (3), with $k a$ for the scattered sound field due to a plane incident wave of unit magnitude for the soft, $\rho c$ and hard spheres. (Color online)

ilarly the frequency limit above which the sphere with a normalized impedance of 100 no longer behaves like a perfectly hard sphere is determined by the denominator of this equation and corresponds to a value of $k a$ of about $100 n$.
TABLE I. The low-frequency limits for the first three nondimensional spherical harmonic coupling coefficients of the scattered sound field due to the incident plane wave, $A_{n}^{s}(k a)$, and those due to a single monopole secondary source on the surface of the sphere, $A_{n}^{c}(k a)$, for a locally reacting sphere which is either soft, i.e. $\zeta \ll k a, \rho c$, i.e. $\zeta=1$, or hard, i.e. $\zeta \gg k a$.

\begin{tabular}{c|c|c|c|c} 
& & $\zeta \ll k a$ & $\zeta=1$ & $\zeta \gg k a$ \\
\hline$A_{n}^{s}(k a)$ & $n=0$ & $-\mathrm{j}(k a)$ & $(k a)^{2}$ & $-\mathrm{j}(k a)^{3} / 3$ \\
& $n=1$ & $-\mathrm{j}(k a)^{3} / 3$ & $\mathrm{j}(k a)^{3} / 6$ & $\mathrm{j}(k a)^{3} / 6$ \\
& $n=2$ & $-\mathrm{j}(k a)^{5} / 45$ & $2 \mathrm{j}(k a)^{5} / 135$ & $2 \mathrm{j}(k a)^{5} / 135$ \\
\hline \multirow{2}{*}{$A_{n}^{c}(k a)$} & $n=0$ & $-\mathrm{j} \zeta(k a)$ & $(k a)^{2}$ & $(k a)^{2}$ \\
& $n=1$ & $-\mathrm{j} \zeta(k a)^{2}$ & $(k a)^{3} / 2$ & $(k a)^{3} / 2$ \\
& $n=2$ & $-\mathrm{j} \zeta(k a)^{3} / 3$ & $(k a)^{4} / 9$ & $(k a)^{4} / 9$ \\
\hline
\end{tabular}

\section{SOUND FIELD DUE TO A MONOPOLE ON THE SUR- FACE OF THE SPHERE}

In this section, we assume that the secondary sources used for active control are monopoles on the surface of the sphere. The pressure due to such a source acting as a control source, of strength $q_{c}$ at an angle of $\theta_{c}, \varphi_{c}$, on the surface of a locally reacting sphere having a normalized surface impedance $\zeta$ can also be expressed in terms of spherical harmonics, as

$$
p_{c}(r, \theta, \varphi)=q_{c} \sum_{n=0}^{\infty} \sum_{m=-n}^{n} B_{n m} h_{n}(k r) Y_{n}^{m}(\theta, \varphi)
$$

where

$$
B_{n m}=\frac{\rho c}{a^{2}} \bar{Y}_{n}^{m}\left(\theta_{c} \varphi_{c}\right) A_{n}^{c}(k a)
$$

and

$$
A_{n}^{c}(k a)=\frac{\zeta}{h_{n}(k a)+\mathrm{j} \zeta h_{n}^{\prime}(k a)},
$$

where $A_{n}^{c}$ is another dimensionless function of $k a$. It is clear from equation (13) that the pressure generated by a monopole placed on a perfectly soft sphere, with $\zeta=0$, would be zero, which motivates the choice of $\zeta=0.01$ for this study.

Figure 4 shows the variation of $A_{n}^{c}(k a)$, in equation (13), with $k a$. If the monopole is on the same or the opposite side of the sphere to the incident wave, this placement ensures that only the $m=0$ term is non-zero, so that the primary and secondary fields have matching contributing components. The variation of the $A_{0}^{c}$ coefficients at higher values of $k a$ for the soft and the hard sphere are relatively smooth when compared with the scattered coefficients for the incident field in Figure 3. For values of $k a$ much less than unity, the $A_{n}^{c}$ coefficients can again be obtained from the small-argument expansions of the Hankel functions to give, for $n=0$

$$
\lim _{k a \ll 1} A_{0}^{c}=(k a)^{2} \frac{\mathrm{j} \zeta}{\mathrm{j} \zeta-k a}\left[1+O\left(k^{2} a^{2}\right)\right],
$$


and for $n \geq 1$

$$
\lim _{k a \ll 1} A_{n}^{c}=\frac{(k a)^{(n+1)}}{(2 n-1) ! !} \frac{\zeta k a}{\zeta(n+1)+\mathrm{j} k a}\left[1+O\left(k^{2} a^{2}\right)\right] .
$$

The low-frequency coefficients for $n=0, n=1$ and $n=2$ are also given in Table I for the soft, $\rho c$, and the hard sphere. For values of $k a$ smaller than unity there is a clear hierarchy in the amplitudes of both the scattered field, Figure 3, and the field due to a secondary source on the surface of the sphere, Figure 4.

\section{ACTIVE CONTROL OF SCATTERED SOUND POWER USING SECONDARY SOURCES ON THE SURFACE OF THE SPHERE}

If $L$ monopole sources on the surface of the sphere are used as secondary sources, the complex amplitude of the $n, m$-th spherical harmonic of the sound field in equation (1), is now due to both the scattered field and the secondary sources and is given by

$$
a_{n m}=a_{n m}^{s}+\sum_{l=1}^{L} B_{n m}^{l} q_{c}^{l}
$$

where $a_{n m}^{s}$ is the amplitude of the spherical harmonic due to the incident wave, as in equation $(2), q_{c}^{l}$ is the complex secondary source strength of the $l$-th secondary source and $B_{n m}^{l}$ is the coupling of the $l$-th secondary source into the $n, m$-th spherical harmonic, as in equation (12).

Assuming that the sum over $n$ in equation (8) only runs over $N$ terms, there are, in general, $(N+1)^{2}$ complex $a_{n m}$ coefficients, and these can be arranged as a vector

$$
\mathbf{a}=\left[a_{00}, a_{1-1}, a_{10}, a_{11}, \cdots, a_{N-N}, \cdots, a_{N 0}, \cdots, a_{N N}\right]^{T} .
$$

The vector of spherical harmonic coefficients due to both the scattered and the control sources can thus be written as

$$
\mathbf{a}=\mathbf{a}_{s}+\mathbf{B} \mathbf{q}_{c}
$$

where $\mathbf{a}_{s}$ is the $(N+1)^{2} \times 1$ vector of complex spherical harmonics due to the scattered wave, $\mathbf{B}$ is the $(N+1)^{2} \times L$ matrix of the complex $B_{n m}^{l}$ coefficients for each secondary source and $\mathbf{q}_{c}$ is the $L \times 1$ vector of complex secondary source strengths.

The normalized scattered power in equation (7) can be written, for the truncated series, using this vector notation as

$$
\Pi_{c}=\frac{1}{\pi(k a)^{2}\left|p_{i}\right|^{2}} \mathbf{a}^{\mathrm{H}} \mathbf{a}
$$

where the superscript $H$ denotes the Hermitian, complex conjugate, transpose. Substituting the equation for the vector of spherical harmonic coefficients into that for the normalized scattered power, this can be written as a standard Hermitian quadratic function of $\mathbf{q}_{c}{ }^{3}$, which has a unique global minimum if the vector of secondary source strengths is given by

$$
\mathbf{q}_{\text {opt }}=-\left[\mathbf{B}^{H} \mathbf{B}\right]^{-1} \mathbf{B}^{\mathrm{H}} \mathbf{a}_{s},
$$

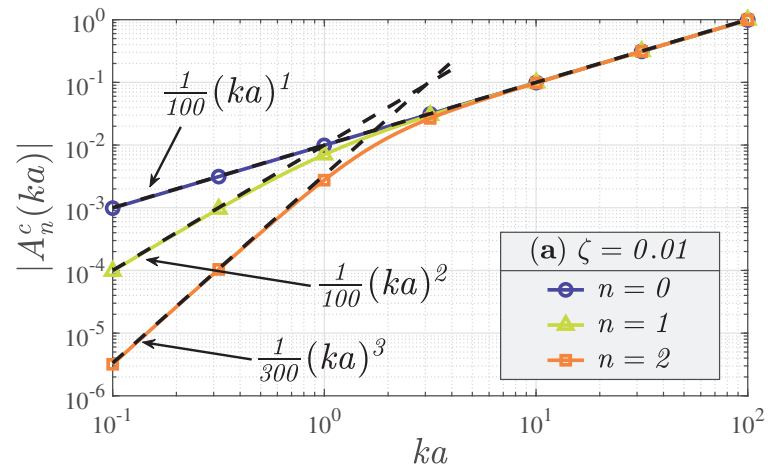

(a)

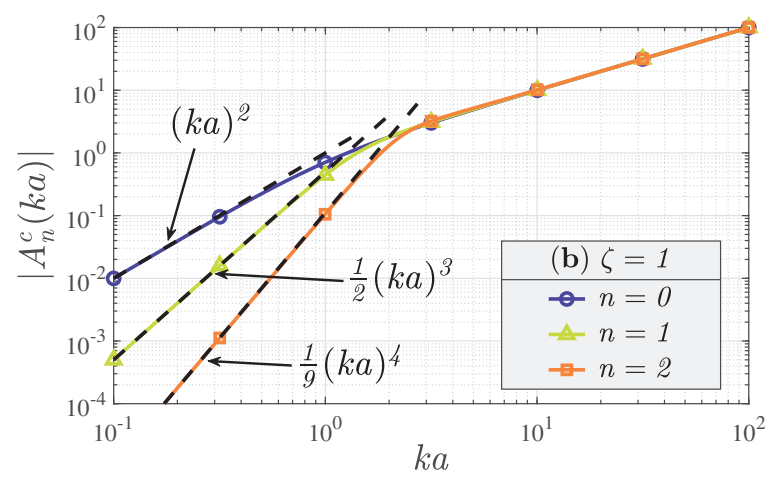

(b)

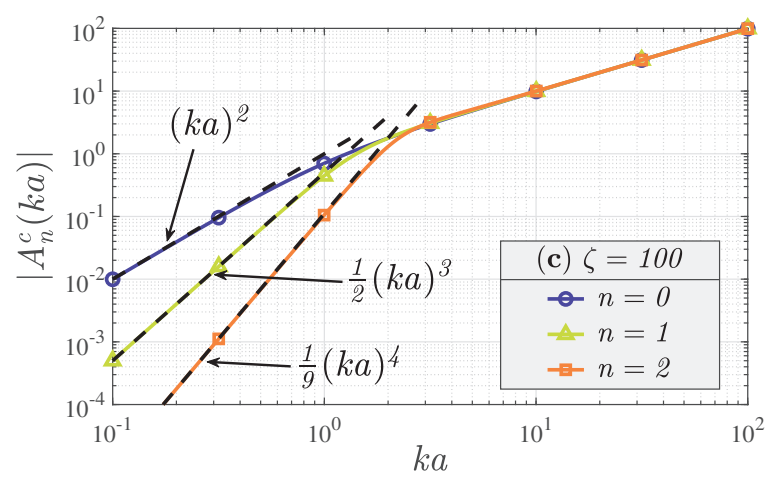

(c)

FIG. 4. The variation of the magnitude of the nondimensional spherical harmonic amplitudes due to a secondary source on the surface of the sphere, $A_{n}^{c}(k a)$ in equation (13), with $k a$ for the soft, $\rho c$, and hard spheres. (Color online)

assuming that $(N+1)^{2}>L$ and that $\mathbf{B}^{\mathrm{H}} \mathbf{B}$ is positive definite. Figure 5 shows the magnitude of the secondary source strength, normalized as described below, as a function of $k a$, calculated using this formulation when a single secondary source, positioned on the opposite side of the sphere to the incident plane wave, is used to optimally control the scattered power. Figure 6 shows the results of such active control in terms of the normalized scattered power for the soft, $\rho c$, and hard spheres without control, as in Figure 1, and after control with a single 
secondary source on the opposite side of the sphere to the incident wave, and also after control with two secondary sources on either side of the sphere along the axis of propagation for the incident wave, as shown in Figure 7. If the incident plane wave and the secondary sources are aligned along the $\mathrm{z}$ axis in this way, only the $m=0$ terms contribute to the pressure field, which simplifies the formulation.

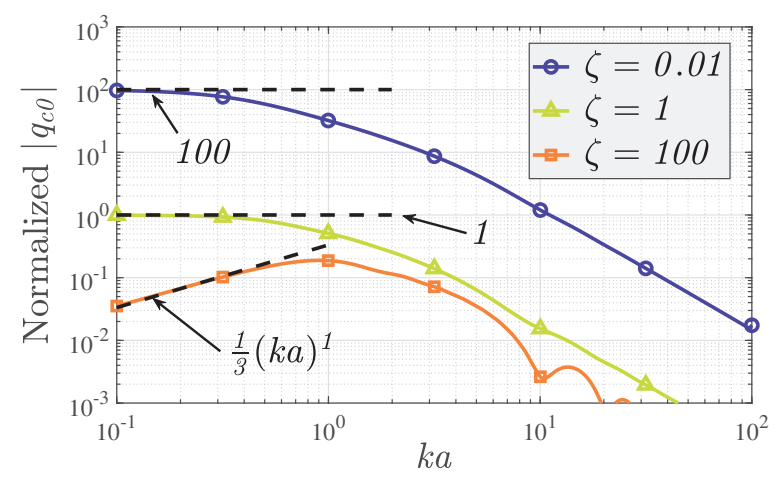

FIG. 5. Magnitude of the normalized volume velocity for the optimised single secondary source used to minimize the scattered sound power from the soft, $\rho c$, and hard sphere. (Color online)

The $n=0$ term in the spherical harmonic expansion of the scattered field is dominant at low frequencies for the soft and the $\rho c$ sphere, as shown in Figure 3(a) and $3(\mathrm{~b})$, and a single secondary source also dominantly couples into the $n=0$ term, as shown in Figure 4 (a) and 4(b). The action of the secondary source in minimising equation (19) will thus be almost the same as cancelling the total $n=0$ field. The total field for $n=0$ with a single secondary source can be written as a specific case of equation (16), as

$$
a_{00}=a_{00}^{s}+B_{00} q_{c},
$$

which can be set to zero if $q_{c}$ is equal to

$$
q_{c 0}=-a_{00}^{s} / B_{00}
$$

Using equations (2) and (12), and since $\bar{Y}_{0}^{0}\left(\theta_{i}, \varphi_{i}\right)$ is equal to $\bar{Y}_{0}^{0}\left(\theta_{c}, \varphi_{c}\right)$ in this case, this can be written as

$$
q_{c 0}=-\frac{4 \pi a^{2} p_{i}}{\rho c} \frac{A_{0}^{s}(k a)}{A_{0}^{c}(k a)} .
$$

The normalized secondary source strength plotted in Figure 5 is given by $q_{c}$ divided by $4 \pi a^{2} p_{i} / \rho c$, and using this normalisation,

$$
q_{c 0} \frac{\rho c}{4 \pi a^{2} p_{i}}=\frac{A_{0}^{s}(k a)}{A_{0}^{c}(k a)} .
$$

Using the results for $A_{0}^{i}(k a)$ and $A_{0}^{c}(k a)$ in Table I, the low frequency normalized secondary source strength can

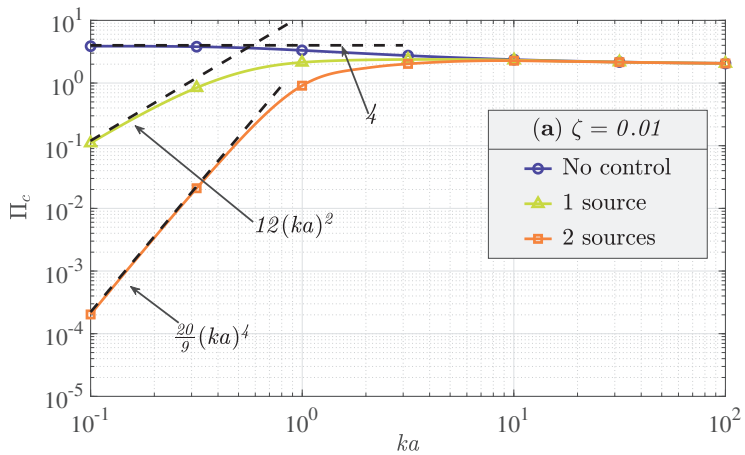

(a)

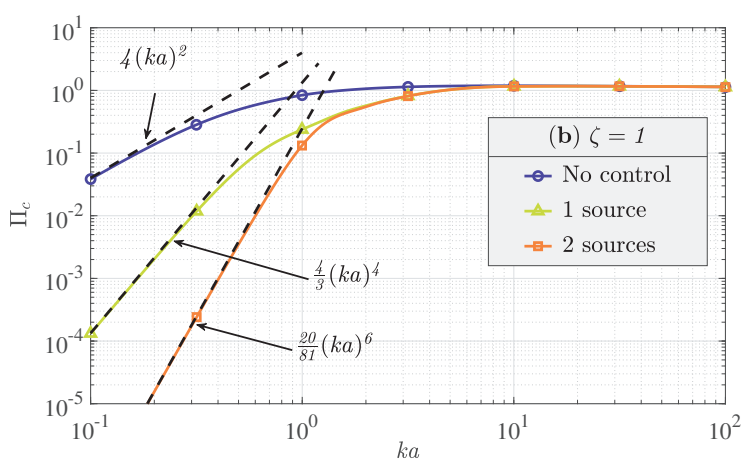

(b)

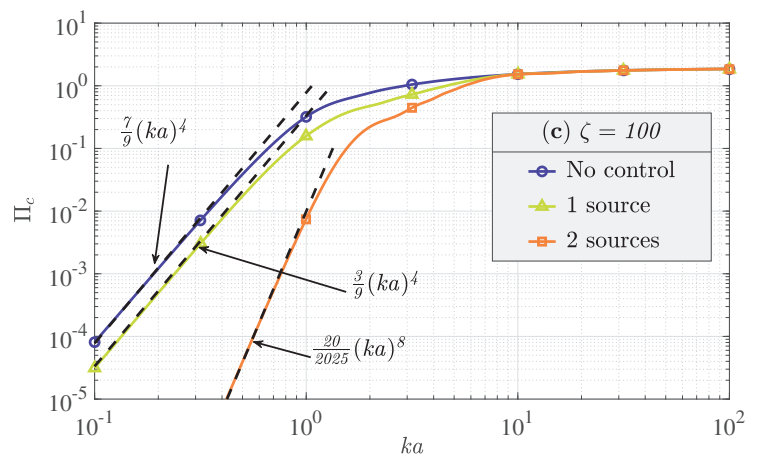

(c)

FIG. 6. The normalized scattered sound power as a function of $k a$ for the soft (a), $\rho c$ (b) and hard (c) spheres, with no active control and with active control using 1 or 2 secondary sources, as shown in Figure 7. (Color online)

be shown to be unity for the $\rho c$ sphere and 100 for the soft sphere with $\zeta=0.01$, as seen in Figure 5 .

The situation is slightly more complicated for the hard sphere since both the $n=0$ and the $n=1$ terms in the scattered field are of similar magnitude, as shown in Figure 3(c). Using equation (8) and the corresponding terms in Table I, the normalized scattered power is found to be $\frac{7}{9}(k a)^{4}$ at low frequencies, with a $\frac{4}{9}(k a)^{4}$ contribution from the $n=0$ terms and a $\frac{3}{9}(k a)^{4}$ contribution from the $n=1$ terms, in agreement with the analysis of $\mathrm{Lamb}^{23}$. At low frequencies, however, a single secondary 


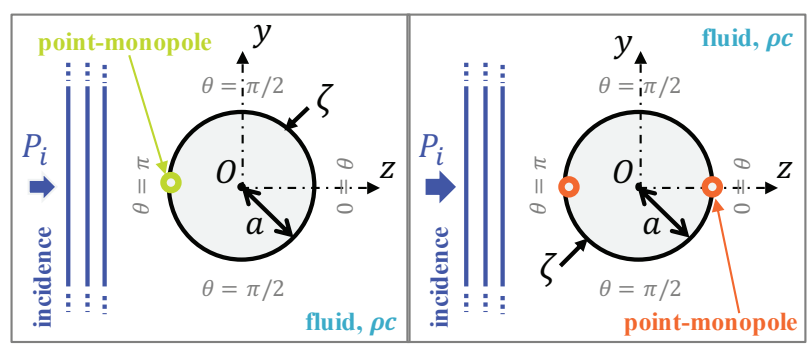

FIG. 7. The positions of the 1 or 2 secondary sources on the sphere relative to the incident plane wave. (Color online)

source will couple much more efficiently into the $n=0$ term in the spherical harmonic expansion rather than the $n=1$ term, as shown in Figure 4(c). Thus, the action of the secondary source in minimising equation (19) is again found to be almost the same as cancelling the $n=0$ total field, as above, so that the normalized secondary source strength is again given by equation (24), which in this case is equal to $\frac{1}{3}(k a)^{4}$, as seen in Figure 5 . The normalized sound power after control is thus equal to $\frac{3}{9}(k a)^{4}$, which is due to the $n=1$ term, and so only $3.7 \mathrm{~dB}$ of attenuation can be achieved with a single secondary source on the surface of the hard sphere, as shown in Figure $6(\mathrm{c})$.

So, in all the cases with a single secondary source, the sound field after control is dominated by the $n=1$ spherical harmonic term, which can be written as

$$
a_{10}=a_{10}^{s}+B_{10} q_{c}
$$

but if $q_{c}$ is given by equation (22), then this becomes

$$
a_{10}=a_{10}^{s}-\frac{B_{10}}{B_{00}} a_{00}^{s}
$$

so that the non-dimensional spherical harmonic amplitudes for the total $n=1$ field can be written for the secondary source on the opposite side to the incident wave as

$$
A_{1}(k a)=A_{1}^{s}(k a)-\mathrm{j} \frac{A_{1}^{c}(k a)}{A_{0}^{c}(k a)} A_{0}^{s}(k a),
$$

where the factor of $\mathrm{j}$ comes from the $\mathrm{j}^{n}$ term in the definition of $a_{n m}^{s}$ in equation (2). Using the low frequency expansion for these quantities in Table I for the soft sphere, $A_{1}(k a)$ is found to be dominated by the $A_{1}^{c}(k a) A_{0}^{s}(k a) / A_{0}^{c}(k a)$ term in equation (27), which is equal to $(k a)^{2}$, rather than the $A_{1}^{s}(k a)$ term, which is equal to $-\mathrm{j}(k a)^{3} / 3$. Using equation (8) again, the normalized power after control at low frequencies is thus predicted to be $12(\mathrm{ka})^{2}$ for the soft sphere, as shown in Figure 6(a). For the $\rho c$ sphere, however, $A_{1}^{s}(k a)$ is equal to $\mathrm{j}(k a)^{3} / 6$ and $A_{1}^{c}(k a) A_{0}^{s}(k a) / A_{0}^{c}(k a)$ is equal to $(k a)^{3} / 2$, so both of these terms contribute significantly to $A_{1}(k a)$ and the normalized power after control is predicted to be $4(k a)^{4} / 3$ at low frequencies, as shown in Figure 6(b).
When two secondary sources are used, one is positioned on the same side of the sphere as the incident wave and one is on the opposite side, as shown in Figure 7. At low frequencies, each of these sources will predominantly couple into the $n=0$ and $n=1$ spherical harmonics, regardless of $\zeta$, as shown by the results in Figure 4 . Since at low frequencies the $n=0$ and the $n=1$ terms in the original scattered field also dominate the $n=2$ term for all values of $\zeta$, as shown in Figure 3, the action of the two secondary sources in minimizing equation (19) at low frequencies will be similar to cancelling the total field due to both the $n=0$ and the $n=1$ spherical harmonics.

A similar analysis to that above can be performed for the residual sound field after control with two secondary sources, in this case in terms of the sum of the two source strengths that dominate both the $n=0$ spherical harmonic, which is cancelled, and the $n=2$ harmonic, which is the residual. The normalized $n=2$ spherical harmonic, after cancellation of the $n=0$ and $n=1$ terms, can then be written as

$$
A_{2}(k a)=A_{2}^{s}(k a)+\frac{A_{2}^{c}(k a)}{A_{0}^{c}(k a)} A_{0}^{s}(k a) .
$$

Using the low frequency approximations in Table I for the individual terms on the right-hand side of equation (28), for the various cases, the normalized power after control is predicted to be $20(k a)^{4} / 9$ for the soft sphere, $20(k a)^{6} / 81$ for the $\rho c$ sphere and $4(k a)^{8} / 405$ for the hard sphere, which are in good agreement with the low frequency results in Figure 6.

Figure 8 shows the low-frequency directivity of the scattered field, before and after active control with the 1 or 2 secondary sources above. The scattering directivity before control is almost omni-directional for the soft and $\rho c$ spheres, since the $n=0$, monopole, term is dominant in the scattering, whereas for the hard sphere it has a more directional form, since both the $n=0$ and $n=1$, monopole and dipole, terms are significant. After control with a single secondary source, the $n=0$ term is suppressed and the residual $n=1$ term gives an almost dipole directivity in all cases. The backscattered pressure, at $\theta=\pi$, is more significantly reduced for the $\rho c$ sphere than for either the soft or hard spheres, which partially reflects the greater level of reduction in the scattered power for the $\rho c$ sphere at this frequency, corresponding to $k a=0.1$, as seen in Figure 6. When two secondary sources are used for control the scattered field is significantly reduced for all angles in every case, and is dominated by the quadrapole-like directivity of the residual $n=2$ term.

It is difficult to physically interpret the results if distributions of secondary sources that are not aligned along the $z$ axis are used, partially because the complex spherical harmonics for $n=1$ and different values of $m$ do not correspond to dipoles aligned along the three Cartesian axes. Nevertheless, it is still interesting to use the theory described above to calculate the attenuation that can be obtained with a larger number of secondary sources. Figure 9 , for example, shows the percentage reduction in the 


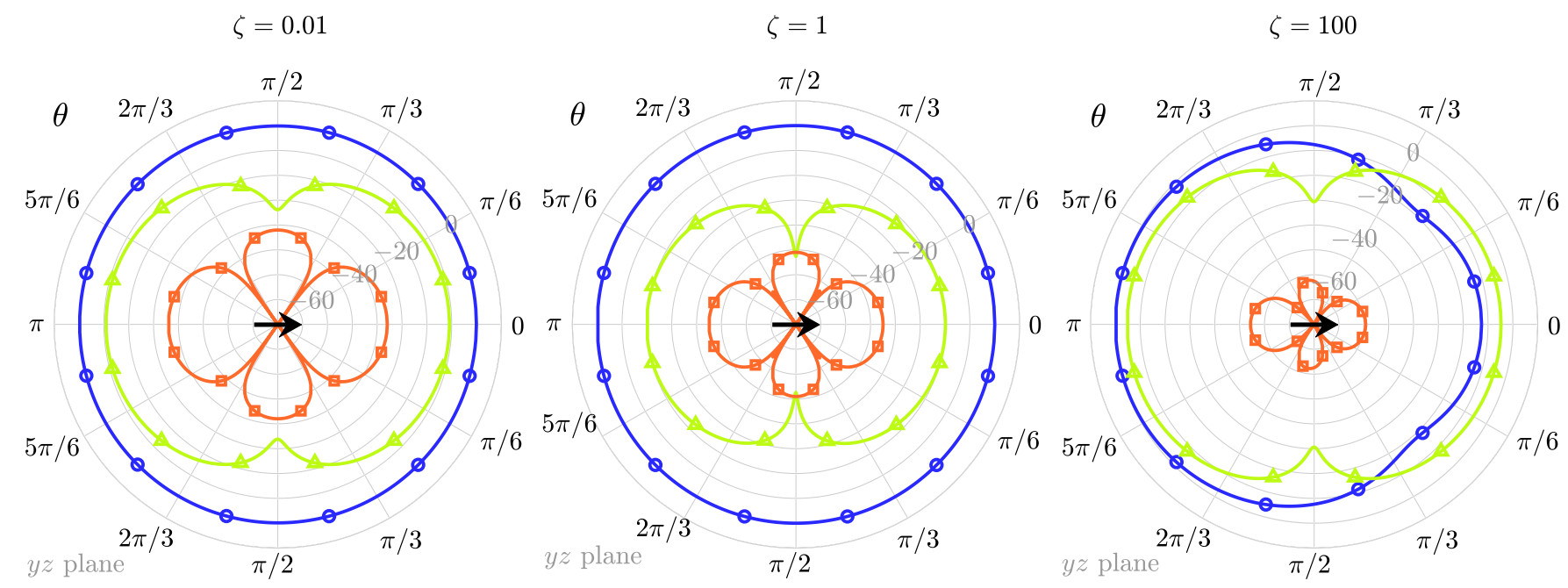

No control

1 source

-2 sources

FIG. 8. The directivity of the scattered field for the soft, $\rho c$ and hard sphere at a normalized frequency of $k a=0.1$ with no control (blue solid) and after active control using 1 (green dashed) or 2 (orange dot-dash) secondary sources. The backscattered pressure before control has been taken as the $0 \mathrm{~dB}$ reference in each case. (Color online).

scattered sound power for the $\rho c$ sphere as a function of $k a$ and the number of secondary sources, which were arranged in a sunflower pattern, as used in ${ }^{9}$. In these computations, the matrix $\mathbf{B}^{H} \mathbf{B}$ was regularized by a small factor if its condition number exceeded $10^{6}$. The number of secondary sources required for a $90 \%$ attenuation, corresponding to a $10 \mathrm{~dB}$ reduction in scattered power, is approximately given by $(k a+1)^{2}$, which is known to be the number of sources required to accurately reproduce a given sound field up to a normalized frequency of $k a^{24}$. It is found that with a large number of sources, very similar results are obtained for the hard and soft spheres, so that in this case the attenuation in the scattered power is relatively independent of the surface impedance of the scattering sphere.

\section{ACTIVE CONTROL WITH SECONDARY SOURCES AWAY FROM THE SURFACE OF THE SPHERE}

The active control of acoustic scattering with secondary sources that are not on the surface of the sphere has been considered by a number of authors, such as Vasquez et $\mathrm{al}^{5}$, Norris et $\mathrm{al}^{6}$, Eggler et $\mathrm{al}^{11}$ and Liu et $\mathrm{al}^{10}$, although Cheer ${ }^{9}$ found that, for a hard sphere, this was less effective than when they were positioned on the surface of the sphere.

The same formulation for active control as in equations (16) to (20) can also be used in the case where the secondary sources are not on the surface of the sphere, except that the non-dimensional term in the coupling be-

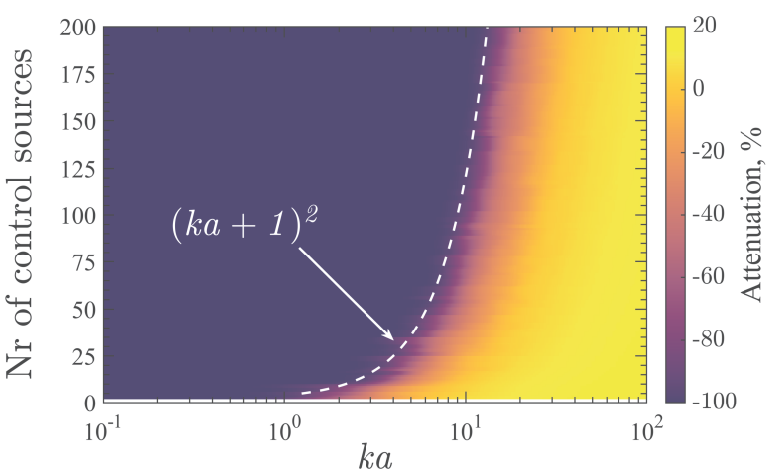

FIG. 9. The percentage reduction in the scattered sound power for the $\rho c$ sphere, as a function of the number of secondary sources, which were arranged in a sunflower pattern, and $k a$. The number of secondary sources required for an $90 \%$ attenuation, corresponding to a $10 \mathrm{~dB}$ reduction, is approximately given by $(k a+1)^{2}$. (Color online)

tween the secondary sources and the spherical harmonics, $A_{n}^{c}(k a)$, is given in this case by the formula

$$
A_{n}^{c}\left(k r^{\prime}\right)=\left[j_{n}\left(k r^{\prime}\right)-A_{n m}^{s}(k a) h_{n}\left(k r^{\prime}\right)\right](k a)^{2},
$$

Where $r^{\prime}$ is the distance from the secondary source to the centre of the sphere, so that equation (29) reduces to equation (13) in the case when $r^{\prime}$ is equal to $a$. The first term in equation (29) can be interpreted as the direct 
field, due to the secondary source in the absence of the scattering sphere, while the second term is due to the scattering of the sound field from the secondary source by the sphere. Figure 10 shows the normalized scattered sound power after control with 200 secondary monopole sources uniformly distributed over a spherical surface of radius $r^{\prime}$ centred on a scattering $\rho c$ sphere of radius $a$, for different values of $r^{\prime}$. As the secondary sources are moved away from the surface of the scattering sphere, the active control performance, at $k a=10$ for example, is very significantly reduced. Similar results to those in Figure 10 are also obtained for the hard sphere, as noted by Cheer $^{9}$, and also for the soft sphere, again indicating that as the number of sources increases, the performance becomes less dependent on the surface impedance.

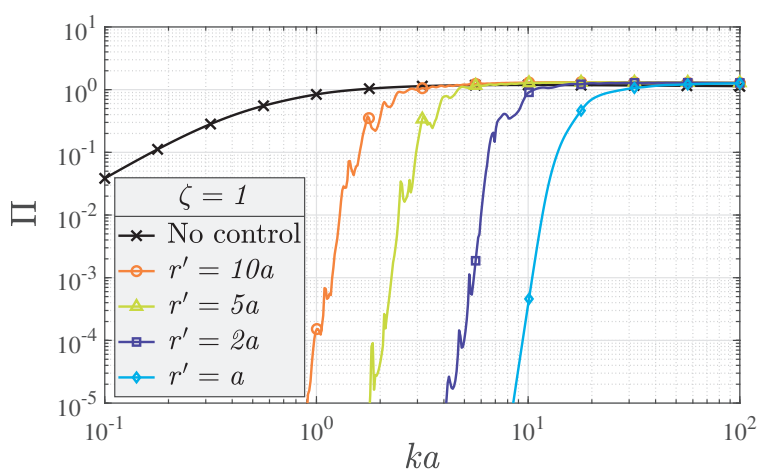

FIG. 10. The normalized scattered power for the $\rho c$ sphere as a function of $k a$ before control and after control with 200 secondary sources placed at different distances, $r^{\prime}$, away from the surface of the sphere. (Color online)

The way that the secondary source couples into the $n=0$ spherical harmonic at low frequencies is found to be relatively independent of its distance from the surface of the sphere. In contrast, the secondary source couples much more efficiently into the higher-order spherical harmonics as it is moved away from the surface of the sphere. This behaviour can be understood by taking the low frequency approximation to each of the terms in equation (29) to give, for the $\rho c$ sphere with $n \geq 1$,

$$
\begin{aligned}
& \lim _{k a \ll 1} A_{n}^{c}\left(k r^{\prime}\right)= \\
& (k a)^{2}\left(k r^{\prime}\right)^{n} \frac{2^{n} n !}{(2 n) !(n+1)}\left[1-\frac{(2 n+1) ! !}{(n+1)}\left(\frac{a}{r^{\prime}}\right)^{2 n+1}\right] .
\end{aligned}
$$

By examining the second term in the square brackets of equation (29), it is clear that if $r^{\prime}>a$ then this term will be small for a sufficiently large value of $n$, so that the first, direct field term will dominate. At low frequencies, and for a sufficiently large value of $n$, the ratio of the coupling term for a source at a distance of $r^{\prime}$ from the surface of the sphere to that with a source on the surface is thus

$$
\lim _{k a \ll 1} \frac{A_{n}^{c}\left(k r^{\prime}\right)}{A_{n}^{c}(k a)}=\left(\frac{r^{\prime}}{a}\right)^{n} .
$$

As the secondary source is moved away from the surface of the sphere, it thus couples more efficiently into the spherical harmonics with a higher value of $n$. The scattered field to be controlled, however, remains dominated by the lower order terms, as shown in Figure 3. So, in controlling these lower order modes the secondary sources will increasingly excite the higher order spherical harmonics as they are moved away from the surface of the sphere, which will degrade the control performance. This is similar to the control spillover effect seen in the active control of modal systems, such as vibration in structures ${ }^{25,26}$, where the secondary sources can excite higher order, and previously poorly excited, structural modes when they are used to attenuate the effects of lower order structural modes.

To a first approximation, however, the guideline used above for the number of sources needed for effective active control being $L=(k a+1)^{2}$ can be generalized for sources away from the surface so that the required number of sources is $L=\left(k r^{\prime}+1\right)^{2}$. Control will thus be effective, for larger vales of $k a$, up to a normalized frequency of

$$
k a=\frac{a}{r^{\prime}} \sqrt{L},
$$

where $L$ is the number of secondary sources, which gives a reasonable prediction of the results in Figure 10.

\section{CONCLUSIONS}

The spherical harmonic analysis is reviewed for the sound field scattered by a sphere with a uniform locally reacting surface impedance, when subject to an incident plane wave. A similar analysis is then presented for the sound field generated by a monopole on the surface of such a sphere, which can be used as a secondary source for active control. The acoustic power scattered by the sphere with a number of such monopoles used as secondary sources is proportional to the sum of squared amplitudes of the spherical harmonics due to both the incident plane wave and the secondary sources. The scattered sound power is thus a quadratic function of the complex secondary source strengths and so has a unique global minimum for a set of secondary source strengths that can be calculated analytically. The results of such calculations are presented in terms of the normalized scattered power, defined as that relative to the sound power associated with the incident wave, the normalized surface impedance and the normalized frequency. The problem of sensing the scattered field in a practical active control system is not considered here, since following the hierarchical design approach for active control systems suggested by Hansen et $\mathrm{al}^{7}$ and Elliott ${ }^{27}$, the physical capabilities of the source, as considered here, is the first issue to be addressed in the analysis of any active control problem, followed by the sensing strategy and then the control system design.

The resulting reductions in the scattered sound power are more pronounced for low frequencies than for high frequencies, as expected, and depend on the num- 
ber of secondary sources. It is found that for a soft sphere, with surface acoustic impedance much less than the characteristic impedance in the surrounding fluid, the scattered sound power is dominated by the zeroth order spherical harmonic and can be very significantly reduced at low frequencies by a single monopole. Additional attenuations can be achieved with two secondary sources aligned along the axis of the incident wave. Similar attenuations in the scattered sound power are observed for a sphere whose impedance is equal to the characteristic impedance of the surrounding fluid, although the magnitude of the scattered power before control is rather smaller in this case.

When the sphere has a surface impedance that is large compared to the fluid characteristic impedance, even less sound power is scattered before control. In this case, at least two secondary sources are required to significantly attenuate the scattered sound power at low frequencies, since both the zero and the first order spherical harmonics contribute significantly to the scattered sound power before control.

Reductions in the scattered sound power can be achieved at higher frequencies only by using a larger number of secondary sources. The number of sources required on the surface of the sphere to achieve a given level of control at a normalized frequency of $k a$ is approximately given by $(k a+1)^{2}$, and hence the square of the frequency, when $k a$ is much greater than 1 , so that the secondary sources are spaced about half a wavelength apart on the surface of the sphere.

It is also found that the active control performance degrades as the secondary sources are moved away from the surface of the sphere. This had previously been observed for the hard sphere, but is generalized here to include spheres with other surface impedances, and is shown to be associated with increased control spillover into higher order spherical harmonics

Though the scattered sound power has been formulated in terms of the spherical harmonics for the scattering body considered here, similar formulations can be used for scattering objects of other shapes. Morse and Feschbach $^{20}$ for example list the 11 coordinates systems in which the acoustic wave equation has separable solutions, and an expansion analogous to that using spherical harmonics can be defined for several of these cases. More generally, the sound radiation from a body of any shape in any acoustic environment can be expressed in terms of the amplitudes of a set of velocity distributions that radiate sound independently from each other, which are known as the radiation modes ${ }^{28-30}$. In fact, the radiation modes for the sphere are the spherical harmonics, as noted by Cunefare et $\mathrm{al}^{31}$. The analysis presented here is simplified by the fact that the shapes of the radiation modes are independent of frequency in the case of the sphere, whereas in the general case the shapes of the radiation modes become weakly dependent on frequency as the wavelength becomes comparable to the size of the object. The sound power scattered by any obstacle can thus be expressed in terms of the sum of the squares of the complex amplitudes of these radiation modes, which are linearly dependent on the secondary source strengths. For scattering bodies of other shapes, a similar formulation to that described above can thus be used to calculate the effect of active control on the scattered sound.

\section{APPENDIX A:}

The spherical wave generated by a monopole source of strength $q$ in free space, can be expressed with the current time-frequency convention as

$$
p(\mathbf{r})=\mathrm{j} k \rho c q \frac{e^{-\mathrm{j} k\left|\mathbf{r}-\mathbf{r}^{\prime}\right|}}{4 \pi\left|\mathbf{r}-\mathbf{r}^{\prime}\right|}, \quad \mathbf{r} \neq \mathbf{r}^{\prime},
$$

where $\mathbf{r}$ is the position vector of the evaluation point in space, $(r, \theta, \varphi)$ and $\mathbf{r}^{\prime}$ is the position vector for the location of the source, $\left(r^{\prime}, \theta_{s}, \varphi_{s}\right)$. After some manipulation $^{18}$, this can be formulated in terms of spherical harmonics as

$$
\begin{aligned}
& p(\mathbf{r})= \\
& k^{2} \rho c q \sum_{n=0}^{\infty} \sum_{m=-n}^{n} j_{n}\left(k r_{<}\right) h_{n}\left(k r_{>}\right) Y_{n}^{m}(\theta, \varphi) \bar{Y}_{n}^{m}\left(\theta_{s}, \varphi_{s}\right) .
\end{aligned}
$$

where $r_{<}=\min \left(|\mathbf{r}|,\left|\mathbf{r}^{\prime}\right|\right)$ and $r_{>}=\max \left(|\mathbf{r}|,\left|\mathbf{r}^{\prime}\right|\right)$. $Y_{n}^{m}(\theta, \varphi)$ are complex spherical harmonics and $h_{n}\left(k r_{>}\right)$ is the complex spherical Hankel function of the second kind, due to the complex frequency convention used here. It should be noted that the Condon-Shortley convention has been used for the phase of spherical harmonics.

Let a monopole primary source with acoustic strength $q_{i}$, generating the incident field, be situated a distance $r^{\prime}$ away from a locally reacting sphere of radius $a$, centred at the origin of the coordinate system. So that $r^{\prime} \geq a$ and $r \geq a$. This situation represents a scattering problem, where the incident field is given by equation (A2). For example, the plan incident wave for a remote source, $k r^{\prime} \gg 1$, becomes

$$
p_{i}(r)=4 \pi p_{i} \sum_{n=0}^{\infty} \sum_{m=-n}^{n}(\mathrm{j})^{n} j_{n}(k a) Y_{n}^{m}(\theta, \varphi) \bar{Y}_{n}^{m}\left(\theta_{i}, \varphi_{i}\right) .
$$

The scattered pressure field, $p_{s}(\mathbf{r})$, can be represented by the exterior solution to the Helmholtz equation ${ }^{18}$ as

$$
p_{s}(\mathbf{r})=\sum_{n=0}^{\infty} \sum_{m=-n}^{n} C_{n m} h_{n}(k r) Y_{n}^{m}\left(\theta_{s}, \varphi_{s}\right)
$$

where $C_{n m}$ are coefficients to be evaluated. The radial pressure gradients of the incident and scattered fields are given, respectively, by

$$
\frac{\partial p_{i}}{\partial r}(\mathbf{r})=k^{3} \rho c q_{i} \sum_{n=0}^{\infty} \sum_{m=-n}^{n} j_{n}^{\prime}(k r) h_{n}\left(k r^{\prime}\right) Y_{n}^{m}(\theta, \varphi) \bar{Y}_{n}^{m}\left(\theta_{s}, \varphi_{s}\right)
$$


where the prime denotes differentiation with respect to the argument and

$$
\frac{\partial p_{s}}{\partial r}(\mathbf{r})=k \sum_{n=0}^{\infty} \sum_{m=-n}^{n} C_{n m} h_{n}^{\prime}(k r) Y_{n}^{m}\left(\theta_{s}, \varphi_{s}\right) .
$$

For a sphere with a locally reacting normalized impedance $\zeta$, the boundary condition is

$$
\left.\left(\mathrm{j} k p_{t}(\mathbf{r})-\zeta \frac{\partial p_{t}(\mathbf{r})}{\partial r}\right)\right|_{r=a}=0,
$$

where $p_{t}(\mathbf{r})=p_{i}(\mathbf{r})+p_{s}(\mathbf{r})$ is the total pressure. Combining equations (A1) to (A6) with this boundary condition, and evaluating for $r=a$ using the orthogonality of the spherical harmonics, it is found that

$$
\begin{aligned}
C_{n m} & =-k^{2} \rho c q_{i} h_{n}\left(k r^{\prime}\right) \frac{j_{n}(k a)+j \zeta j_{n}^{\prime}(k a)}{h_{n}(k a)+j \zeta h_{n}^{\prime}(k a)} \bar{Y}_{n}^{m}\left(\theta_{s}, \varphi_{s}\right) \\
& =-k^{2} \rho c q_{i} h_{n}\left(k r^{\prime}\right) A_{n}^{i}(k a) \bar{Y}_{n}^{m}\left(\theta_{s}, \varphi_{s}\right),
\end{aligned}
$$

with $A_{n}^{s}(k a)$ defined as in equation (3). The scattered pressure field in (A4) is thus given by

$$
\begin{gathered}
p_{s}(\mathbf{r})=-k^{2} \rho c q_{i} \sum_{n=0}^{\infty} \sum_{m=-n}^{n} A_{n}^{i}(k a) h_{n}(k r) h_{n}\left(k r^{\prime}\right) \cdots \\
Y_{n}^{m}(\theta, \varphi) \bar{Y}_{n}^{m}\left(\theta_{s}, \varphi_{s}\right) .
\end{gathered}
$$

If $r^{\prime} \gg a$, then the incident field becomes a plane wave, with an incident pressure given by equation (A1), and taking the large argument limit for $h_{n}\left(k r^{\prime}\right)$, equation (A9) then reduces to equations (1) and (2) in the main text.

If, on the other hand, a monopole on the surface of the sphere, of source strength $q_{c}$, is used as a secondary source, then the total pressure field, for $r>r^{\prime}$ is given by the sum of the direct field in equation (A2) and the scattered field in equation (A9) as

$$
\begin{gathered}
p_{t}(\mathbf{r})=k^{2} \rho c q_{c} \sum_{n=0}^{\infty} \sum_{m=-n}^{n}\left[j_{n}(k a)-A_{n}^{i}(k a) h_{n}(k a)\right] \cdots \\
h_{n}(k r) Y_{n}^{m}(\theta, \varphi) \bar{Y}_{n}^{m}\left(\theta_{c}, \varphi_{c}\right),
\end{gathered}
$$

and equation (A10) then reduces to equations (11) and (12) for the resulting pressure in the main text.

\section{Acknowledgment}

The authors are grateful for discussion with Dr John Smith from the Defence, Science and Technology Laboratory, UK, who are supporting a $\mathrm{PhD}$ studentship for Mihai Orita.

\footnotetext{
${ }^{1}$ R. O. Duda and W. L. Martens, "Range dependence of the response of a spherical head model," The Journal of the Acoustical Society of America 104(5), 3048-3058 (1998).

${ }^{2}$ H. Xu, R. Xia, J. Li, and Y. Yan, "An improved free-field crosstalk cancellation method based on the spherical head model," Applied Acoustics 123, 47-54 (2017).
}

${ }^{3}$ P. A. Nelson and S. J. Elliott, Active control of sound (Academic press, 1991).

${ }^{4}$ E. Friot, R. Guillermin, and M. Winninger, "Active control of scattered acoustic radiation: a real-time implementation for a three-dimensional object," Acta acustica united with acustica 92(2), 278-288 (2006).

${ }^{5}$ F. G. Vasquez, G. W. Milton, and D. Onofrei, "Exterior cloaking with active sources in two dimensional acoustics," Wave Motion 48(6), 515-524 (2011).

${ }^{6}$ A. N. Norris, F. A. Amirkulova, and W. J. Parnell, "Source amplitudes for active exterior cloaking," Inverse Problems 28(10), 105002 (2012).

${ }^{7}$ C. Hansen, S. Snyder, X. Qiu, L. Brooks, and D. Moreau, Active control of noise and vibration (CRC press, 2012).

${ }^{8}$ E. Friot and C. Bordier, "Real-time active suppression of scattered acoustic radiation," Journal of sound and vibration $\mathbf{2 7 8}(3)$, 563-580 (2004).

${ }^{9} \mathrm{~J}$. Cheer, "Active control of scattered acoustic fields: Cancellation, reproduction and cloaking," The Journal of the Acoustical Society of America 140(3), 1502-1512 (2016).

${ }^{10}$ J. Liu, X. Wang, M. Wu, and J. Yang, "An active control strategy for the scattered sound field control of a rigid sphere," The Journal of the Acoustical Society of America 144(1), EL52-EL58 (2018).

${ }^{11}$ D. Eggler, H. Chung, F. Montiel, J. Pan, and N. Kessissoglou, "Active noise cloaking of 2D cylindrical shells," Wave Motion 87, 106-122 (2019).

${ }^{12}$ M. A. Poletti, "Three-dimensional surround sound systems based on spherical harmonics," Journal of the Audio Engineering Society 53(11), 1004-1025 (2005).

${ }^{13}$ M. Orita, S. Elliott, and J. Cheer, "Spherical harmonic formulations for passive and active control of acoustic scattering around the sphere," The Journal of the Acoustical Society of America 144(3), 1757-1757 (2018).

${ }^{14} \mathrm{~J}$. W. S. Rayleigh, "Investigation of the disturbance produced by a spherical obstacle on the waves of sound," Proceedings of the London Mathematical Society s1-4(1), 253-283 (1871).

${ }^{15}$ J. W. S. Rayleigh, The theory of sound, 1896 ed. (Dover, New York, 1945).

${ }^{16}$ O. A. Godin, "Rayleigh scattering of a spherical sound wave," The Journal of the Acoustical Society of America 133(2), 709720 (2013).

${ }^{17}$ Y. Mao, J. Cai, Y. Gu, and D. Qi, "Direct evaluation of acousticintensity vector field around an impedance scattering body," AIAA Journal 53(5), 1362-1371 (2014).

${ }^{18}$ E. G. Williams, Fourier acoustics: sound radiation and nearfield acoustical holography (Elsevier, 1999).

${ }^{19}$ B. Rafaely, Fundamentals of spherical array processing, Vol. 8 (Springer, 2015).

${ }^{20}$ P. M. Morse and H. Feshbach, "Methods of theoretical physics," American Journal of Physics 22(6), 410-413 (1954).

${ }^{21} \mathrm{~S}$. Temkin, Elements of acoustics (Wiley New York, 1981).

${ }^{22} \mathrm{~S}$. Brown, S. Wang, and D. Sen, "Analysis of the sphericalwave truncation error for spherical harmonic soundfield expansions," in 2012 IEEE International Conference on Acoustics, Speech and Signal Processing (ICASSP), IEEE (2012), pp. 5-8.

${ }^{23} \mathrm{H}$. Lamb, The dynamical theory of sound, $1960 \mathrm{ed}$. (Dover, 1925).

${ }^{24}$ D. B. Ward and T. D. Abhayapala, "Reproduction of a planewave sound field using an array of loudspeakers," IEEE Transactions on speech and audio processing 9(6), 697-707 (2001).

${ }^{25}$ L. Meirovitch, H. Baruh, and H. Oz, "A comparison of control techniques for large flexible systems," Journal of Guidance, Control, and Dynamics 6(4), 302-310 (1983).

${ }^{26}$ C. C. Fuller, S. J. Elliott, and P. A. Nelson, Active control of vibration (Academic Press, 1996).

${ }^{27}$ S. J. Elliott, Signal processing for active control (Elsevier, 2000). 
${ }^{28} \mathrm{G}$. V. Borgiotti, "The power radiated by a vibrating body in an acoustic fluid and its determination from boundary measurements," The Journal of the Acoustical Society of America 88(4), 1884-1893 (1990).

${ }^{29}$ D. M. Photiadis, "The relationship of singular value decomposition to wave-vector filtering in sound radiation problems," The Journal of the Acoustical Society of America 88(2), 1152-1159 (1990).

${ }^{30}$ S. J. Elliott and M. E. Johnson, "Radiation modes and the active control of sound power," The Journal of the Acoustical Society of America 94(4), 2194-2204 (1993).

${ }^{31}$ K. A. Cunefare, M. N. Currey, M. E. Johnson, and S. J. Elliott, "The radiation efficiency grouping of free-space acoustic radiation modes," The Journal of the Acoustical Society of America 109(1), 203-215 (2001). 